\title{
Transplantation of the Liver
}

\author{
Charles W. Putnam, M.D. ${ }^{*}$ and Thomas E. Starzl, M.D., Ph.D. ${ }^{\dagger}$ \\ Departments of Surgery, Veterans Administration Hospital and University of Colorado Medical \\ Center, Denver, Colorado
}

Clinical orthotopic liver transplantation-removal of the entire liver and its replacement with a homograft—was first performed on March 1, 1963 at the University of Colorado. During the next 13 years, approximately 265 such procedures have been performed throughout the world. The University of Colorado series now numbers 113 patients, or about 40 per cent of the total experience. In a recent extensive review, our first 93 cases were analyzed in detail, emphasizing clinical and pathologic correlations. ${ }^{4}$

In this article, we will focus upon certain areas of our experience; our results with this procedure, our present views regarding candidacy for liver replacement, and finally, selected diagnostic and technical problems that are encountered during and after liver transplantation, particularly the problem of secondary jaundice.

\section{SURVIVAL AFTER LIVER TRANSPLANTATION}

Of the 113 patients in the University of Colorado series, 103 were transplanted more than 1 year ago. Of these 103 at risk, 30 (or 29 per cent) have survived for at least 1 year after liver replacement (Table 1).

Since 1968 the 1-year survival figures have been relatively constant from year to year. Despite the very heavy early mortality, one which must be considered unacceptable, many of the patients who have reached the 1-year mark have continued to do well. For example, 15 of the 301 -year survivors are still alive. Fifteen patients have reached the 2-year mark, eight have reached 3 years, six have survived 4 years, and there have been four 5 -year survivors (see Table 1). Our longest survivor, who is now 62/3 years post-transplantation, is living at home, attending school, and has absolutely normal liver function. Two other 5-year survivors are also entirely well, but the fourth one died a few days short of the 6-year mark of chronic rejection and partial biliary obstruction. Of the 151 -year survivors who subsequently died, four have succumbed to recurrent tumor, five to chronic rejection, and two each died of hepatitis, of bile duct obstruction, and of septic complications unrelated to biliary tract problems (Table 2), In retrospect, at least some of these late deaths might have been avoided, particularly those caused by failure of biliary drainage.

\section{CANDIDACY FOR LIVER TRANSPLANTATION}

\section{Influence of Age}

Not surprisingly, older patients have a poorer prognosis after liver transplantation. For example, during the 5-year period 1969-1974, only 13 per cent of recipients over 40 years of age survived a year or more after operation versus 42 per cent for those 40 years or younger (Table 3). The much increased mortality in these older recipients has largely been the

\footnotetext{
*Assistant Professor of Surgery, University of Colorado Medical Center

$\dagger$ Professor and Chairman, Department of Surgery, University of Colorado Medical Center
} 
consequence of pUlmonary or other infections, difficulties with maintaining nutrition, or neurologic complications.

\section{Influence of Hepatic Disease}

Transplantation for Hepatic Malignancy-Early in our experience, nonresectable hepatic malignancies were a popular indication for liver replacement. In the University of Colorado series, 12 patients have been transplanted for nonresectable hepatoma, cholangiocarcinoma, or hemangioendothelial sarcoma. There were seven early deaths not related to malignancy. The other five patients, however, all died of recurrence, 87 to 432 days after transplantation. Usually, widespread pulmonary metastases developed and, typically, the grafted livers were also involved by malignancy. Because of this tendency to develop recurrence, we believe that patients with large nonresectable malignant tumors are questionable candidates for liver replacement. Exceptions to this policy of exclusion have been made when the tumor is an incidental finding in a liver afflicted with another disease. For example, one of our patients transplanted for biliary atresia had a small hepatoma in the excised organ. She has absolutely no evidence of recurrence 62/3 years after transplantation and, in fact, is our longest survivor.

Another kind of malignancy which we initially thought might be a good indication for liver transplantation is the so-called "Klatskin tumor." 2 These small duct cell carcinomas usually arise at the bifurcation of the hepatic ducts and kill by obstruction, rather than by widespread disease (Fig. 1). Of the four patients we have transplanted for this indication, two died early after operation. One of the other two patients is alive and clinically free of tumor 2 years post-transplantation. The other, however, died of recurrent tumor in his transplanted liver 2 years after operation. Thus, we are unsure of the long-term prognosis in such cases but we believe that further trials in younger patients with this malignancy are warranted, since liver replacement is the only procedure with the likelihood of cure. Those patients with Klatskin tumors who are not candidates for transplantation can often be effectively palliated, as has been so well demonstrated by Terblanche. ${ }^{5}$

\section{Benign Hepatic Diseases}

Biliary Atresia: Children with biliary atresia were originally thought to be ideal candidates for liver transplantation; this has not always proved to be so in individual cases. Although these children usually enjoy relatively good health until late in the course of their disease, this advantage has in some patients been more than offset by technical difficulties at the time of transplantation. Congenital anomalies of the hepatic vasculature, which are not uncommon in patients with biliary atresia, have made it difficult or impossible to revascularize the homograft livers in some instances. In other children, technical problems posed by the need to work with tiny vessels contributed to failure. These latter difficulties have been at least partially circumvented by the adoption of microsurgical techniques.

It is worth pointing out that we believe that the Kasai procedure (or porticoenterostomy) for biliary atresia and liver transplantation are complementary and not competitive procedures. Even when bile drainage cannot be achieved, the Kasai procedure does not preclude subsequent transplantation. In those patients with progressive cirrhosis despite the successful establishment of bile flow, the porticoenterostomy has successfully "bought time" for the patient, enabling him to grow and develop, thus making the task of transplantation easier. More importantly, by prolonging survival the period of candidacy for transplantation is lengthened. This is obviously particularly important in these young candidates for transplantation for whom potential donors are few and far between. One of our patients, for example, underwent a Kasai procedure at 4 months of age. At 44 months, a liver biopsy showed severe cirrhosis and the patient was referred for transplantation, which was carried 
out 15 months later. She now has normal liver function, nearly 3 years post-transplantation (Fig. 2).

Alcoholic Cirrhosis: Through the years, our worst results have been in patients with alcoholic cirrhosis. Of ten alcoholics transplanted, only one, a former professional football player, has achieved long-term survival. If the patient has completely repudiated the use of alcohol, there should be no intrinsic contraindication to transplantation. In practice, however, the uncertain course of the disease has usually resulted in consideration for transplantation being delayed until such time as one or more complications has supervened. Thus, these patients are usually not referred until repeated episodes of gastrointestinal bleeding, pneumonitis and other infectious complications, or severe hepatic encephalopathy have occurred, thereby severely jeopardizing the chances for recovery. Improvement in survival after transplantation in this group of patients will therefore require that these patients be considered for the procedure before these complications are present and while the candidate still enjoys relatively good health.

Chronic Aggressive Hepatitis: In contrast, those patients with postnecrotic cirrhosis or chronic aggressive hepatitis have a much more favorable prognosis. These patients are usually younger than those with alcoholic cirrhosis. Moreover, the downhill course of their disease is generally more predictable. Those patients with a negative hepatitis B antigen preoperatively do not seem to have a significant risk of developing recurrent hepatitis postoperatively.

The spectre of recurrent hepatitis looms more ominously if the indication for transplantation is $\mathrm{HB}_{\mathrm{s}} \mathrm{Ag}$ positive hepatitis. One of our patients transplanted for this disease developed acute, antigen-positive hepatitis postoperatively, which progressed to chronic active hepatitis from which she eventually died. In two patients with antigen-positive hepatitis treated subsequently, high titer anti- $\mathrm{HB}_{\mathrm{s}} \mathrm{Ag}$ antiserum was given intra- and postoperatively. Preliminary observations suggest that the antiserum was successful in eliminating antigen remaining after removal of the infected liver. Whether this precaution will prevent the subsequent occurrence of post-transplantation hepatitis can only be answered by future trials, since neither patient survived the early convalescent period.

Inborn Errors of Metabolism: Certain hepatic-based inborn errors of metabolism appear to be especially favorable indications for liver transplantation. We have had experience with two such diseases: Wilson's disease and alpha ${ }_{1}$-antitrypsin deficiency.

In Wilson's disease, there is a congenital defect of copper metabolism, which is frequently accompanied by hepatic cirrhosis. Both patients that we have transplanted for this indication have survived 5 years, although one of them died shortly before the 6-year mark of chronic rejection and bile duct obstruction. In both patients, transplantation of a normal liver cured the metabolic defect. Ceruloplasmin, which was quite low preoperatively, rose to the normal range. Copper metabolism became normal, and there has been no tendency to reaccumulate copper in the homografts. Moreover, the neurologic stigmata of Wilson's disease gradually disappeared.

Alpha $_{1}$-antitrypsin deficiency is a hereditary disease in which the homozygous phenotype, $\mathrm{Pi}^{\mathrm{ZZ}}$, predisposes to congenital infantile cirrhosis and occasionally adult cirrhosis, pulmonary emphysema of early onset in adults and, rarely, both pulmonary and hepatic disease either in childhood or adult life. The $\mathrm{Pi} Z \mathrm{ZZ}$ phenotype is not terribly rare; its incidence is estimated to be about one in 2500 live births, about the same as that of cystic fibrosis. About 20 to 30 per cent of children who have the $\mathrm{Pi}^{\mathrm{ZZ}}$ phenotype will go on to develop cirrhosis. The cause for the cirrhosis is not known, but observations after liver 
transplantation for this disease indicate that the liver is the sole source of the missing alpha 1- $^{-}$ antitrypsin. ${ }^{3}$

We have transplanted a 16-year-old girl with alpha ${ }_{1}$-antitrypsin deficiency. Postoperatively, she developed normal levels of alpha ${ }_{1}$-antitrypsin, adopting the donor phenotype, $\mathrm{Pi}^{\mathrm{MM}}$

(Table 4). Two years after transplantation her graft failed from chronic rejection. The donor for her second transplant by coincidence had the heterozygous $\mathrm{Pi}^{\mathrm{MZ}}$ phenotype and a correspondingly reduced serum alpha ${ }_{1}$-antitrypsin concentration (heterozygotS1s, however, have no predilection to develop liver disease). Following the retransplantation, the recipient's serum again adopted the liver donor's phenotype, this time $\mathrm{Pi}^{\mathrm{MZ}}$ (see Table 4). Although the patient died a month later of pulmonary infectious complications, the validity of the metabolic correction by liver transplantation for this disease was established.

Daloze $^{1}$ has reported the metabolic cure of a third liver-based, inborn error of metabolism, Niemann-Pick disease. Recently we have transplanted a child with tyrosinemia who also had a hepatoma in her cirrhotic liver. Biochemical studies are underway, but this may prove to be a fourth inborn error treatable by liver replacement.

Other Benign Diseases: Successful liver replacement has also been carried out for primary biliary cirrhosis and the Budd-Chiari syndrome. We have not yet successfully transplanted patients for fulminant liver failure from hepatitiS or other toxic agents. A major logistical problem in the latter cases involves finding an appropriate cadaveric organ donor during the very brief time span in which such patients are legitimate candidates for transplantation.

\section{Immunologic Criteria for Donor-Recipient Selection}

Unlike patients with end-stage renal disease, candidates for llver transplantation have no artificial surport system comparable to hemodialysis available to them. Consequently, their period of candidacy for liver transplantation is necessarily shorter, requiring a relaxation of criteria for donor-recipient selection, if they are to be treated at all. Indeed, if the deteriorating condition of the recipient warrants doing so, all ordinary immunologic restrictions may be ignored and an $\mathrm{ABO}$ incompatible or cytotoxic-crossmatch positive organ inserted. Under less pressing circumstances, however, the following guidelines are observed.

ABO Compatibility-The criteria for ABO selection of recipients are identical to those for transplantation of other organs such as the kidney. If these red cell barriers are violated in renal transplantation, hyperacute rejection of the organ is the usual, although not invariable, consequence. This does not appear to be the case after liver transplantation, Seven of our patients have received ABO incompatible organs (Table 5). Hyperacute rejection did not occur in any of these recipients and all but one survived at least a month postoperatively. It is unclear from this experience whether these organs may be more susceptible to late rejection, but it is clear that hyperacute rejection of the human liver is quite uncommon, if it occurs at all.

Cytotoxic Antibody Crossmatches-The presence of preformed antidonor antibodies, as demonstrated by a positive cytotoxic crossmatch, likewise nearly invariably forecasts hyperacute rejection of renal homografts. Again the liver seems quite resistant to this immunologic complication, since placement of a hepatic homograft in this immunologically hostile environment does not seem to cause its immediate rejection and the long-term prognosis of such patients appears no different than those with a negative crossmatch. 
HL-A Tissue Typing-The results of histocompatibility matching in our first 103 cases are given in Table 6. No effort has been made to select HL-A compatible donor-recipient combinations, a policy clearly reflected by the very small number of good matches. With such a skewed spectrum of histocompatibility, meaningful conclusions about the validity of the techniques used cannot be drawn. Nonetheless, there is no definable correlation between the quality of match and survival. Obviously, conclusions about the validity of HL-A matching are far more likely to come from experience with renal transplantation in which there is much greater flexibility of donor-recipient pairing.

\section{TECHNIQUES OF ORTHOTOPIC LIVER TRANSPLANTATION}

The surgical techniques of orthotopic liver transplantation have largely become

standardized. The vena cava is anastomosed to the analogous host vessels above and below the liver (Fig. 3). The portal venous anastomosis is an end-to-end one and the hepatic artery is usually revascularized with the common hepatic artery of the recipient.

The major technical problems have arisen from failure to achieve or maintain satisfactory biliary drainage. Unti 1973 the usual technique for restoring biliary continuity was with cholecystoduodenostomy (Fig. 3A). There were definite pitfalls to this approach. First, in a few cases, technical errors were made at the time of transplantation because of the failure to recognize cystic duct anomalies in the donor. In these instances, the cystic duct was occluded when the common duct was ligated prior to performing cholecystoduodenostomy. Accordingly, operative cholangiography became a routine in the donor room several years ago.

Even though satisfactory biliary drainage was achieved initially by cholecystoenterostomy, this was no guarantee that obstruction might not occur later. When obstruction did occurand it has in about a third of the cases in which the gallbladder was used for anastomosis-it was always at the level of the cystic duct. With obstruction, a syndrome of jaundice, fever, septicemia, and biochemical evidence of cholangitis developed. In the first few cases, the correct diagnosis was not made until autopsy. Subsequently, several cases were diagnosed during life by operative cholangiography. Nevertheless, reoperation was usually too late, the patients dying of septic complications.

In 1973, we adopted a policy of performing transhepatic cholangiography whenever recurrent jaundice appeared after transplantation. For this purpose, we have used the very thin needle developed in Chiba, Japan, the so-called "Chiba" or "skinny" needle. With this technique, the procedure has proved to be quite safe. and immensely helpful.

If obstruction of the cystic duct is diagnosed with transbepatic cholangiography, the patient is promptly reoperated and a secondary anastomosis is performed using the dilated common duct (Figs. $3 C$ and 4). Transhepatic cholangiography is equally valuable if a normal duct system is demonstrated. If the clinical picture suggests rejection, prompt intensification of steroid therapy is made. Needle biopsy of the liver is helpful in confirming the diagnosis, particularly if drug hepatotoxicity appears to be an alternate possibility.

In addition to the adoption of frequent transhepatic cholangiography, a second change has been made with regard to biliary drainage. It gradually became apparent that cholangitis and systemic sepsis were frequently being encountered even without mechanical obstruction. With cholecystoduodenostomy the biliary tract is in direct continuity with the mainstream gastrointestinal tract (see Fig. 3A). By interposing a long, defunctionalized Roux-en-Y limb of jejunum between the biliary tree and the gastrointestinal tract it was hoped that the frequency of hepatic sepsis would thereby be reduced. If cystic duct obstruction occurs, the gallbladder is removed and a choledochojejunbstomy performed (Fig. 3C). 
Despite these improvements, the provision of satisfactory biliary drainage remains the fundamental technological problem in liver transplantation. Certain principles of reconstruction have emerged, however. Ideally, the biliary reconstruction should avoid using the cystic duct with its inherently high risk of obstruction. Secondly, the liver should be protected from the bacterially-contaminated mainstream gastrointestinal tract, either by a defunctionalized Roux-en-Y limb of jejunum or by the sphincter 6f Oddi. And thirdly, radiographic visualization of the biliary tree, either by direct tube cholangiography or by the transhepatic approach, must be obtained if jaundice, cholangitis, or systemic sepsis occurs. Choledochocholedochostomy with T-tube drainage satisfies these criteria, but carries a much higher risk of bile fistula than cholecystoenterostomy (Fig. 3D). Alternatively, choledochojejunostomy may be feasible in some cases, although the small size of the normal donor common duct makes this a difficult primary anastomosis. When it is used, stenting of the anastomosis with a small Silastic catheter allows sampling of the bile, frequent cholangiography, and may reduce the incidence of fistula formation.

It seems clear that no one kind of biliary reconstruction will be satisfactory for all patients and that techniques of reconstruction must be individualized in each case.

\section{THE FUTURE OF LIVER TRANSPLANTATION}

Fifteen years ago, few surgeons or nephrologists would have predicted that renal transplantation would be so phenomenally successful, much less that it would become a socialized form of medical therapy, financed by the Federal Government!

Does the same promise hold true for hepatic transplantation? No longer in its infancy (it has been almost 10 years since the first long-term survivor was treated), liver transplantation as it enters adolescence still faces an uncertain future. The technical problems surrounding this procedure have largely been solved with one glaring exception, the problem of reestablishing biliary drainage. During the last 3 or 4 years, refinements in the surgical techniques of reconstruction, a better appreciation of the role of bacterial contamination, and recently developed diagnostic techniques such as transhepatic cholangiography have all contributed to a better understanding of past failures and provide a road map for future progress. The precise application of these lessons should reduce the early mortality after this procedure. The accurate and early diagnosis of biliary tract problems will also indirectly reduce the incidence of non-graft related septic complications, since "obstruction masquerading as rejection" has frequently led to unnecessary, inopportune, and frequently fatal overimmunosuppression.

Even with the prevention or perfect management of biliary tract complications, a number of grafts-and patients-will continue to be lost from rejection. A greater willingness to accept the need for retransplantation rather than pursuing aggressive immunosuppression may reduce patient mortality just as has this policy following cadaveric renal transplantation. Obviously, the technical obstacles to replacing a hepatic homograft are considerably more formidable than for providing a second renal transplant, but retransplantation may prove to be a more acceptable alternative than the frequently lethal consequences of heavy steroid therapy.

Still, as with transplantation of the kidney, heart, bone marrow, and other organs, real hope for wide clinical applicability rests with an improvement in immunosuppression that would allow cadaveric organs to be transplanted with the relative ease accompanying the transfer of related homografts. 


\section{Acknowledgments}

The work was supported by research grants MRIS 8118-01 and 7227-01 from the Veterans Administration; by Grant Numbers AM-17260 and AM-07772 from the National Institutes of Health; and by Grant Numbers RR-00051 and RR-00069 from the General Clinical Research Centers Program of the Division of Research Resources, National Institutes of Health.

\section{REFERENCES}

1. Daloze P, Corman J, Block P, et al. Enzyme replacement in Niemann-Pick disease by liver homotransplantation. Transplant. Proc. 1975; 7:607.

2. Klatskin G. Adenocarcinoma of the hepatic duct at its bifurcation within the porta hepatitis; an unusual tumor with distinctive clinical and pathological features. Am. J. Med. 1965; 38:241. [PubMed: 14256720]

3. Putnam CW, Porter KA, Peters RL, et al. Liver replacement for alpha 1 -antitrypsin deficiency. Surgery. in press.

4. Starzl TE, Porter KA, Putnam CW, et al. Orthotopic liver transplantation in ninety-three patients. Surg. Gynec. Obstet. 1976; 142:487. [PubMed: 176741]

5. Terblanche J, Saunders SJ, Louw JH. Prolonged palliation in carcinoma of the main hepatic duct junction. Surgery. 1972; 71:720. [PubMed: 4112754] 


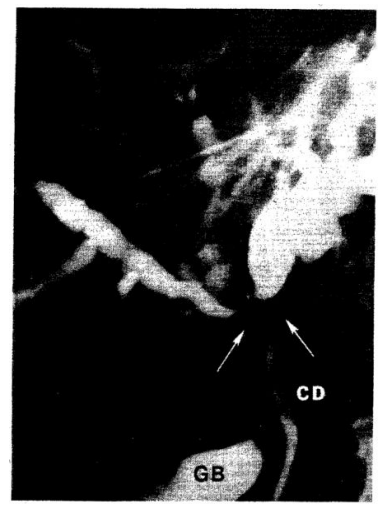

Figure 1.

Preoperative transhepatic cholangiogram of a very small duct cell tumor at the bifurcation of the hepatic ducts (arrows). Liver replacement was successfully performed but the patient died of recurrent tumor 2 years later. 


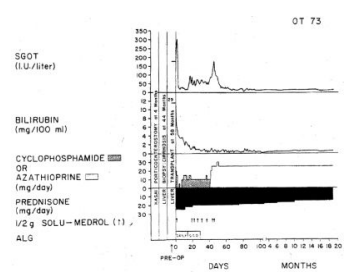

Figure 2.

The course of a child who had a failed Kasai operation but who ultimately was effectively treated with liver replacement. (By pelmission of Josiah Macy, Jr. Foundation, 1976, in press.) 

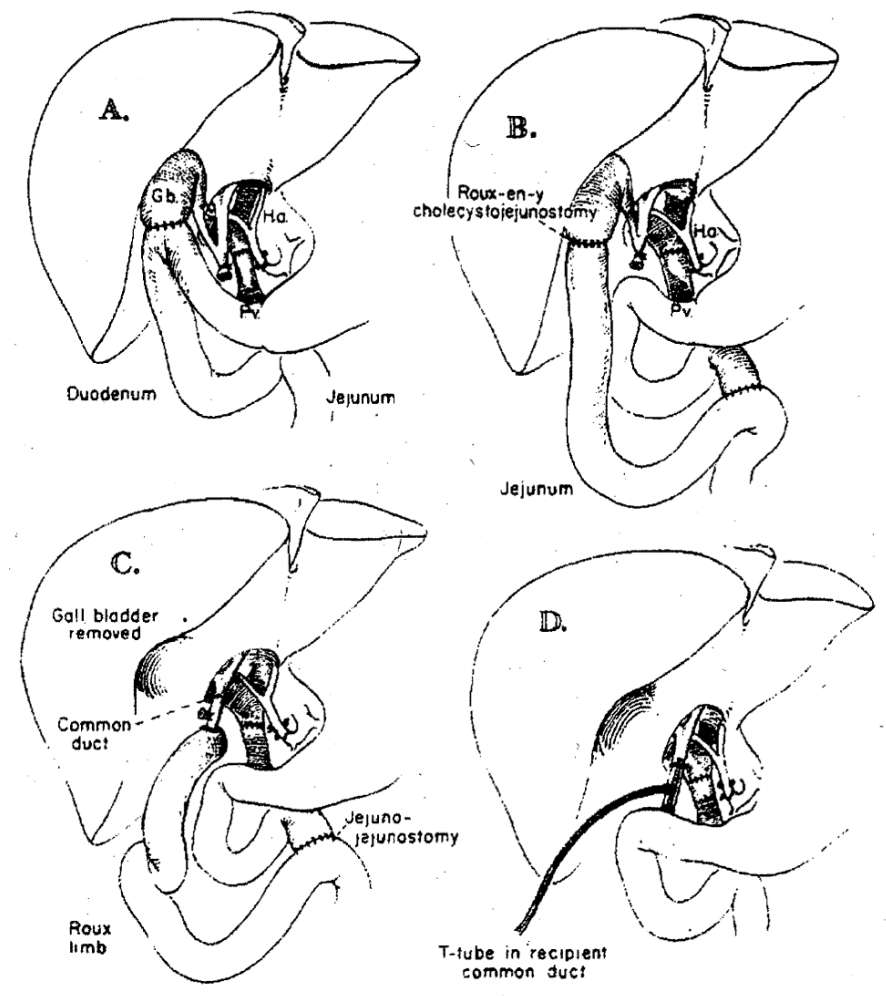

Figure 3.

Techniques of biliary duct reconstruction used in conjunction with liver transplantation: $A$, cholecystoduodenostomy; $B$, cholecystojejunostomy; $C$, choledochojejunostomy after removal of gallbladder; $D$, choledochocholedochostomy. Note that the T-tube is placed, if possible, in the recipient common duct. (Reproduced from Starzl, T. E., Porter, K. A.,

Putnam, C. W., et al.: Orthotopic liver transplantation in 93 patients. Surg. Gynec. Obstet., 142:487,1976. Used with permission.) 

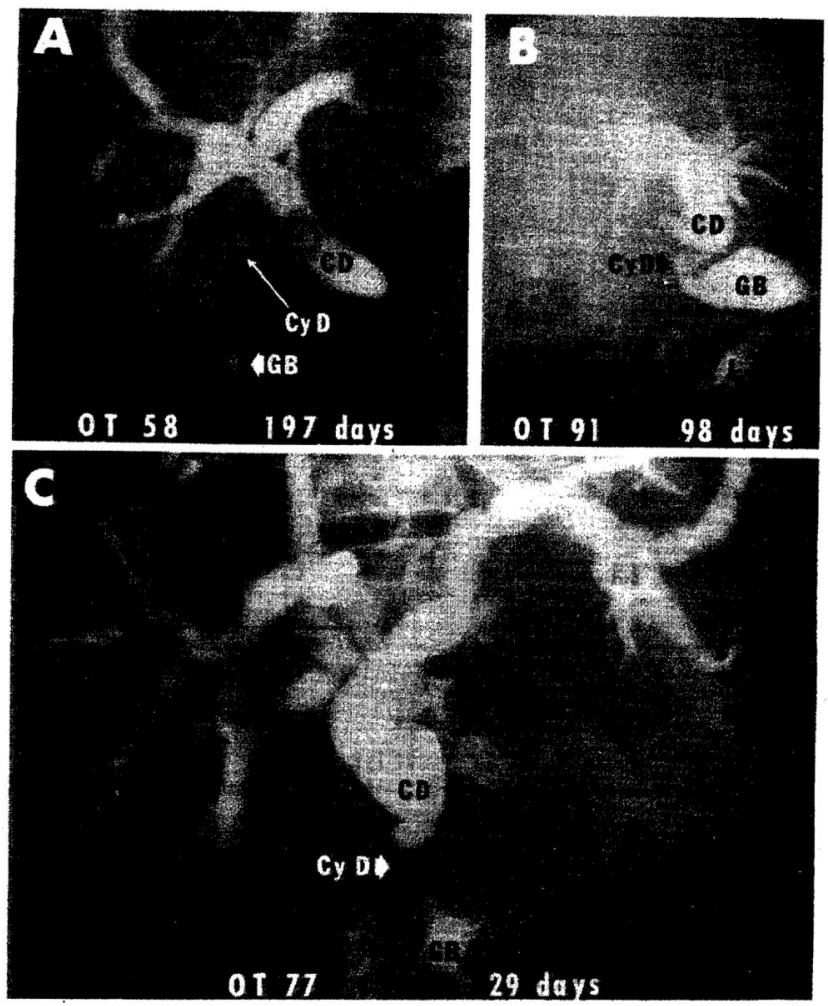

Figure 4.

Transhepatic cholangiography after liver replacement demonstrating cystic duct obstruction after cholecystoenterostomy. A, Original procedure was cholecystoduodenostomy. After this transhepatic cholangiogram, conversion was made to choledochoduodenostomy. At operation, the filling defect near the exit of the cystic duct was found to consist of a chalklike sludge. There was not complete relief of jaundice. When the patient died 13 months after transplantation, the homograft still had intrahepatic evidence of large duct obstruction. $B$, The original reconstruction was with cholecysto-Roux-en-Y-jejunostomy. This was converted to a choledochojejunostomy. The patient is well almost 2 years later. $C$, The original reconstruction was with cholecysto-Roux-en-Y-jejunostomy. This was converted to a choledochojejunostomy. The patient is well about $21 / 2$ years later. ( $C D$, common bile duct: $C y D$, cystic duct; $G B$, gallbladder; $J$, Roux-en-Y limb of jejunum.) 


\section{Table 2}

Present Status of 30 1-Year Survivors

\begin{tabular}{|c|c|c|c|}
\hline Alive $15 / 30$ & 一 & 12 months to 80 months & \\
\hline \multirow[t]{6}{*}{ Dead 15/30 } & 一 & 12 months to 71 months & \\
\hline & & Recurrent cancer & 4 \\
\hline & & Chronic rejection & 5 \\
\hline & & Chronic hepatitis & 2 \\
\hline & & Bile duct obstruction & 2 \\
\hline & & Other infections & 2 \\
\hline
\end{tabular}

Surg Clin North Am. Author manuscript; available in PMC 2011 April 29. 
Table 3

One-Year Survival by Age (1969-1974)

\begin{tabular}{crrc}
\hline $\begin{array}{c}\text { AGE } \\
\text { (YEARS) }\end{array}$ & $\begin{array}{r}\text { NO. } \\
\text { PATIENTS }\end{array}$ & $\begin{array}{r}\text { 1-YEAR } \\
\text { SURVIVAL }\end{array}$ & $\begin{array}{c}\text { PER CENT } \\
\text { SURVIVING }\end{array}$ \\
\hline 0 to 5 & 24 & 9 & 38 per cent \\
6 to 15 & 10 & 3 & 30 per cent \\
16 to 25 & 9 & 6 & 67 per cent \\
26 to 40 & 10 & 4 & 40 per cent \\
$41+$ & 16 & 2 & 13 per cent \\
TOTAL & 69 & 24 & 35 per cent \\
Patient $\leq 40$ years & 53 & 22 & 42 per cent \\
\hline
\end{tabular}




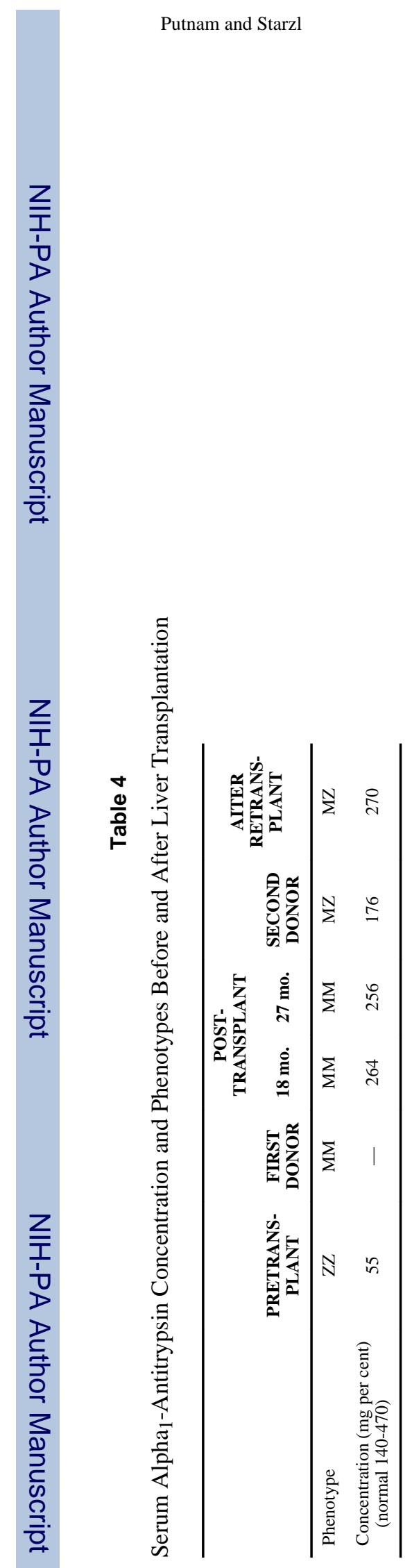

Surg Clin North Am. Author manuscript; available in PMC 2011 April 29. 


\section{Table 6}

HL-A Typing of Primary Hepatic Grafts in 103 Cases

\begin{tabular}{crc}
\hline MATCH $^{*}$ & NUMBER & $\begin{array}{c}\text { SURVIVAL } \\
\text { I YEAR }\end{array}$ \\
\hline A & 1 & $1^{\dagger}$ \\
B & 3 & $1(33$ per cent $)$ \\
C & 15 & $3(20$ per cent $)$ \\
D & 27 & $5(19$ per cent $)$ \\
E & 44 & $16(36$ per cent $)$ \\
F & 6 & $1(17$ per cent $)$ \\
Not done & 7 & $1(14$ per cent $)$ \\
TOTAL & 103 & $27(29$ per cent $)$ \\
\hline
\end{tabular}

A-Match: HL-A identity between recipient and donor.

B-Match: Compatibility between donor and recipient, but fewer antigens determined in the donor.

C-Match:. One antigen incompatible.

D-Match: Two antigens incompatible.

E-Match: Three or four antigens incompatible.

F-Match: ABO violation or positive crossmatch to cytotoxic antibodies.

${ }^{\dagger}$ Retransplanted after 68 days with C-Match graft. Thus, the 1-year survival was due mainly to the second less well matched organ. 